\section{(6) OPEN ACCESS}

\title{
Implications of the concept of minimal risk in research on informed choice in clinical practice
}

\author{
Kyoko Wada, Jeff Nisker
}

Department of Obstetrics and Gynaecology, Schulich School of Medicine \& Dentistry, Western University, London, Ontario, Canada

\section{Correspondence to} Dr Jeff Nisker, Department of Obstetrics and Gynaecology, Schulich School of Medicine \& Dentistry, Children's Health Research Institute, Western University, London Health Sciences Centre, Victoria Hospital, Rm E2-620E, 800 Commissioners Rd. E., London, Ontario, Canada N6A 5W9; jeff.nisker@|hsc.on.ca

Received 9 May 2014 Revised 1 April 2015 Accepted 8 June 2015 Published Online First 24 June 2015

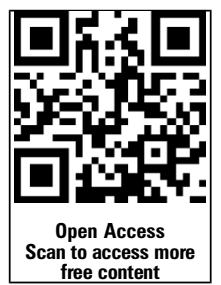

CrossMark

\begin{tabular}{l}
\hline To cite: Wada K, Nisker J. J \\
Med Ethics 2015;41: \\
804-808. \\
\hline
\end{tabular}

\section{ABSTRACT}

The concept of a minimal risk threshold in research, beneath which exception to informed consent and ethics review processes may occur, has been codified for over 30 years in many national research regulations and by the Council for International Organizations of Medical Sciences. Although minimal risk in research constitutes one of the criteria for allowing waiver of informed consent or modification to the consent process and a large body of literature exists, discussion of a minimal risk threshold in clinical practice has not occurred. One reason for lack of discussion may be that implicit consent is accepted for a wide range of routine clinical practices. Extending the role of minimal risk in research to clinical practice might assist clinicians in identifying circumstances for which implicit consent is indeed sufficient and circumstances in which it is not. Further, concepts from minimal risk in research might assist clinicians regarding when information provision in health promotion is required. We begin by reviewing concepts in both minimal risk in research and informed choice in clinical practice. We then explore how a clinical minimal risk concept may clarify recommendations for information provision in clinical practice and support the patient's informed choice regarding therapeutic and diagnostic procedures and also health promotion. Given that clinical practice involves a broad scope of health information, professional practice guidelines on information provision based on the application of the minimal risk threshold in research could be developed to guide clinicians in what information must be provided to their patients.

\section{INTRODUCTION}

The concept of minimal risk in research as a 'sorting threshold' (p.351), ${ }^{1}$ beneath which exception to informed consent and ethics review processes may occur, has been codified for over 30 years in many national research regulations as well as by the Council for International Organizations of Medical Sciences (CIOMS). ${ }^{1-7}$ In regards to informed consent in research, minimal risk constitutes one of the criteria for allowing modification to all or part of the consent process $^{18}$ (45 Code of Federal Regulations (CFR) 46.116, 45 CFR 46.117; TCPS2, Articles 3.7; CIOMS Guidelines 4). In clinical practice, however, there is no concept comparable with minimal risk in research that would provide a low risk threshold for considering waiver of consent or alteration to the consent process. Conversely, acknowledgement of a minimal risk concept in clinical practice could insist that all risks above such a threshold require a formal consent process involving detailed discussion of risk. A minimal risk concept in clinical practice could go beyond discussion of risks of clinical therapies and procedures to include health risks in the patient's lifestyle such as unhealthy diet or environmental chemicals.

A clinician's failure to provide the patient with pertinent information has ethical and potentially legal implications. ${ }^{9}{ }^{10}$ As clinicians are under considerable time constraints in their practice, ${ }^{11}$ a minimal risk concept, such as that well described in clinical research, could be helpful in terms of assuring appropriate information provision to the patient. Although clinical research and practice have different objectives, ${ }^{12}{ }^{13}$ informed consent is an essential ethical requirement in clinical practice as well as in research. ${ }^{9} 13$ Historically, the Nuremberg Code (1947) articulated research ethics requirements. Over time, these requirements influenced clinical medicine (p.4). ${ }^{14}$ Legal proceedings in the 1950 s and 1960s resulted in clinicians acknowledging the significance of obtaining consent upon disclosure of relevant information, both in clinical practice and research (p.515). ${ }^{13}$ Despite these common backgrounds, informed consent is highly regulated in clinical research compared with clinical practice. $^{12} 13 \quad 15$ The differences of regulatory requirements between clinical research and clinical practice may evoke concern that patients may not be sufficiently protected compared with research participants (p.522). ${ }^{13}$

This paper explores the possibility of extending the minimal risk concept in research to information provision in clinical practice. First, we will discuss minimal risk in research regulations regarding informed consent. Second, as there is no minimal risk concept in clinical practice, we will discuss the existing elements and standards of information provision in clinical practice. Finally, we will explore how minimal risk in research may be applied to information provision and informed choice in clinical practice. Acknowledging the existence of a large amount of literature on risk, the risk concept used in this paper will primarily refer to the expectation value of an undesirable event, determined by the probability and severity of outcomes. ${ }^{16}$ We will argue that extending the role of minimal risk in research as a low risk threshold to clinical practice may assist clinicians in understanding their obligation for information provision regarding risks in therapies, diagnostic procedures and lifestyle choices.

\section{MINIMAL RISK IN RESEARCH}

In research regulations regarding human participants, such as the US CFR (45 CFR 46.102(i) $)^{5}$ 
and the Tri-Council Policy Statement of Canada (TCPS2) (Chapter 2.B), ${ }^{7}$ risks may be considered below minimal risk when the likelihood and seriousness of harm or discomfort are comparable with those of daily life. In research regulations, such as the International Ethical Guidelines for Biomedical Research Involving Human Subjects (CIOMS Guidelines) (Guidelines 4 and 9$)^{6}$ as well as in the US CFR (45 CFR 46.102(i)), ${ }^{5}$ risks are considered within minimal risk when the risks are not above the risks of routine clinical (physical and psychological) examinations. Similar concepts of minimal risk are employed across other national and international regulations with some differences $^{1}$ such as Guidelines on Ethics for Medical Research ${ }^{2}$ or Additional Protocol to the Convention on Human Rights and Biomedicine Concerning Biomedical Research. ${ }^{3}$

The risks of daily life or routine clinical examinations as thresholds embedded in the minimal risk in research standard has received much scrutiny, including commentators inquiring whose daily life or routine clinical examinations should be referenced and why risks in daily life or routine clinical examinations should serve the role as a low risk standard. ${ }^{1}{ }^{4}{ }^{17}$ Although these discussions largely focus on research with children in nontherapeutic situations and do not specifically discuss its relevance as a threshold in relation to informed consent, ${ }^{47-19}$ they may provide insight into how a low risk standard could be determined.

Two interpretations of minimal risk are discussed widely in the literature. ${ }^{1}{ }^{4}{ }^{17}$ The absolute interpretation of minimal risk provides the same standard for everyone by referring to risks in daily life or routine clinical examination of an average healthy person. ${ }^{14} 17$ The relative interpretation of minimal risk leads to different standards across persons depending on what kind of risk that particular person lives with. ${ }^{14}{ }^{17}$ The relative interpretation has been criticised as it may label high-risk procedures as minimal-risk procedures if the participant lives with high daily risk such as living in an unsafe neighbourhood or routinely requiring a high-risk clinical intervention. ${ }^{18}{ }^{80}$ However, those who support the relative interpretation indicate that it can consider cultural and societal norms ${ }^{18}$ and that potential abuse may be addressed by ethics review committee (ERC) members who have fiduciary duties to participants, ${ }^{18}$ which is stipulated, for example, in the TCPS2 (p.23, Chapter 2-B). Nonetheless, leaving much to ERC discretion may potentially lead to exploitation of participants ${ }^{80}$ particularly when ERC members are influenced by the value of research or the prestige of the investigators. ${ }^{19}$

Next, it is a normative choice to determine that risks in daily life or routine clinical examinations are relevant to serve the roles of minimal risk. ${ }^{18}$ The daily life standard has been criticised for involving higher risks compared with the routine clinical examination standard. ${ }^{14}{ }^{17}$ Daily life involves a variety of harms, ranging from very trivial to highly serious with various possibilities. ${ }^{1} 4 \quad 8 \quad 17$ By contrast, routine clinical examinations for healthy persons may involve very low physical risks ${ }^{1} 417$ although these examinations may involve potentially high psychosocial risk due to personal information collected prior to such examinations if not kept confidential. ${ }^{12} 20$

Comparing minimal risk standards across research regulations, Kopelman ${ }^{1}$ concludes that the absolute interpretation of the routine clinical examination standard is more justifiable than others as it sufficiently excludes high risks. She articulates that some concern regarding potentially serious psychosocial harm due to inappropriate disclosure of private information may be extremely low due to clinicians' compliance with fiduciary duties. ${ }^{1}$ Kopelman ${ }^{1}$ also proposes that the routine clinical examination standard should rather be understood as risks involved in these examinations per se and not information collected alongside these examinations. ${ }^{1}$ Similarly, Resnik $^{8}$ proposes to employ only the routine clinical examination standard. $\mathrm{He}$ argues that rather than flexibility, the clarity of the standard and consistency in its application should take priority to secure fairness or justice. On the other hand, Freedman $e t$ al ${ }^{18}$ argue that the daily life standard is morally justifiable as (1) research risks are substitutive due to people's exposure to daily risks while not participating in research and (2) daily risks are socially acceptable. ${ }^{18}$ The first point is criticised as daily risks are usually associated with some purpose or benefits whereas research does not guarantee any benefit to the participant. ${ }^{4}$ Indeed, the total benefit gained from the same time frame may likely decrease particularly in research without potential therapeutic benefits to participants. The second point is also criticised as what is socially acceptable may not necessarily be clear ${ }^{1}$ and daily risks are not always socially acceptable but simply unavoidable. ${ }^{4}$ Perhaps, a socially acceptable standard is what may be justifiable.

\section{INFORMED CONSENT AND MINIMAL RISK IN RESEARCH}

Informed consent, independent ethics review and special protection to vulnerable populations are among the basic requirements in conducting ethically sound research. ${ }^{21}$ Among these requirements, informed consent enables a person to protect oneself through the opportunity to receive information and to give or not give consent. ${ }^{92}$ Although a person has a basic right not to be researched upon without informed consent, ${ }^{23}$ alteration to the informed consent process in minimal risk research can occur if other criteria are satisfied, such as ERC approval, very low likelihood of a particular adverse consequence and impracticality of obtaining consent (45 CFR 46.116(c); TCPS2, Article 3.7; CIOMS, Commentary on Guideline 4). Further regarding information provision, research regulations stipulate extensive risk disclosure, that is, reasonably foreseeable risk (TPCS2, Article 3.2, 45CFR46.116(a,2), CIOMS Guideline 5(9)), discomfort (45CFR46.116(a,2), CIOMS Guideline 5(9)) and inconvenience (CIOMS Guideline 5(9)). However, there are debates over the extensiveness of information provision depending on the research procedures and context, which largely revolve around information that is meaningful for the participant's decision-making. ${ }^{24-28}$

\section{INFORMED CONSENT IN CLINICAL PRACTICE}

Informed consent is an essential part of standard of care in clinical practice. ${ }^{29}$ Unlike clinical research, there has been little focus in clinical practice on below what risk level a potential complication of a therapeutic or diagnostic procedure need not be discussed with a patient. However, circumstances seem to exist which allow clinicians to proceed with certain clinical procedures with implicit consent, suggesting that conditions for not requiring formal consent or risk disclosure may exist. ${ }^{9} 14$ 30-35

\section{Elements of information provision in clinical practice}

As part of information provision regarding clinical therapies and procedures, the elements of disclosure must take into consideration (1) the patient's diagnosis, (2) the nature of the procedure, (3) alternatives, (4) risks and benefits of the proposed procedure and the alternatives and (5) prognosis with and without the therapy or procedure. ${ }^{9} 1033$ 36-38 These elements of disclosure commonly appear in professional guidelines for clinicians ${ }^{33} 3738$ as part of the information which need to be communicated to the patient in considering diagnostic or therapeutic procedures. 
The law has given much emphasis on the risk component of disclosure and this has considerably influenced clinical practice. ${ }^{9}$ According to court cases, risks that (1) are so obvious, (2) are known to the patient, (3) are very unlikely or (4) could not be known to the clinician at the time of disclosure can be exempt from disclosure. ${ }^{10}$ Nevertheless, legal obligations differ across jurisdictions ${ }^{9}$ and no clear legal rule exists to specify the level or types of risks that need not be disclosed. ${ }^{10}$ The criteria developed from the court cases seem to refer to the familiarity of the risk to the patient and the clinical community rather than to the risk level. For a clinician's liability protection, Appelbaum et al ${ }^{9}$ recommend that clinicians should disclose risks that are (1) relatively minor but likely to occur and (2) extremely unlikely but very serious-even if not deemed an obligation-as there is no guarantee that all agents involved will agree on what risk qualifies for non-disclosure (p.55). Their recommendation includes consideration to the frequency and seriousness of risk, ${ }^{9}$ which may be similar to how minimal risk in research is framed but obviously less concrete.

\section{Standards of information provision in clinical practice}

Standards of disclosure have evolved largely through legal proceedings determining whether clinician's disclosure was adequate. ${ }^{39}$ Mainly, three standards of disclosure are recognised: the professional, reasonable patient (person) and subjective standards. ${ }^{9} 103539$ Relevance of disclosure is determined by what a typical agent in each category would perceive as sufficient disclosure. ${ }^{9} 39$ The influence of legal cases shifted focus from the traditional professional standard to the reasonable patient standard. ${ }^{13} 36$ The President's Commission for the Study of Ethical Problems in Medicine and Biomedical and Behavioral Research ${ }^{40}$ indicates that the reasonable patient standard can identify a relevant amount of information to support the patient's decision-making and protect clinicians from the patient's retrospection.

The subjective standard arises from the intention of information provision in professional practice guidelines to be bidirectional and multipurpose, ${ }^{33} 37^{41}$ with the appreciation that interactive flows of information are required for the clinician's understanding of the patient's values and concerns that are required for a good clinical relationship and meaningful informed choice. ${ }^{42}$ Although the subjective standard appears ethically preferable to the reasonable patient standard as what is considered 'reasonable' may differ across persons, ${ }^{9} 1036$ full reliance on the subjective standard may not be feasible both legally and ethically as it may be too demanding for clinicians and be open to the patient's retrospection in court. ${ }^{9}$

\section{Explicit versus implied informed consent in clinical practice}

Consent can be implied by persons' action, inaction, words and silence under particular contexts where they can reasonably be expected to know what they are consenting to. ${ }^{9} 35$ The concept of 'general consent' is a practical device to authorise 'routine' procedures that all patients commonly go through upon being seen at healthcare institutions without specific consent. ${ }^{35}$ A general consent form is usually signed when the person registers as a patient. ${ }^{31} 35$ The concept of general consent assumes the patient's familiarity with the practice or procedure and its context allows implied consent for that practice or procedure. $^{9} 35$

Explicit and specific consent is deemed necessary for invasive procedures and intrusive uses of information and tissues to reduce ambiguities regarding the procedures for which consent was obtained. ${ }^{14}$ Some documents by healthcare institutions refer to paradigmatic examples to describe when explicit consent is required. ${ }^{31}{ }^{32}$ For example, a patient brochure by the American Hospital Association ${ }^{31}$ states that a separate consent besides general consent may be required for procedures such as surgery or experimental treatment. The Mayo Clinic Medical Manual ${ }^{32}$ indicates that procedures that are more invasive than a simple intravenous line require explicit consent. By contrast, the World Medical Association ${ }^{33}$ provides a simple rule, stipulating that procedures involving risk or more than mild discomfort require informed consent. General Medical Council of $\mathrm{UK}^{30}$ states that written consent is preferred when complex information or significant risk is involved, investigational components are included, or the consequence of the procedure may affect the patient's social or personal life. As described in these documents, risks or potentially serious outcomes of the procedure call for explicit consent. ${ }^{30-33}$ However, rules such as more than mild discomfort or significant risk may be rather abstract. Also, good examples could be misleading without a specific rule since examples cannot be exhaustive. Significantly, the Canadian Medical Association ${ }^{34}$ states that procedures for the benefit of others require explicit consent, which may share some aspects with the research context where participants are not guaranteed to receive direct benefits.

\section{APPLICATION OF MINIMAL RISK IN RESEARCH TO CLINICAL CONTEXTS}

Although concepts of information provision and consent in the research context are not always analogous to the clinical context, there are important learnings from the large literature on minimal risk in research that may be useful to clinical practice well beyond the circumstances in which implied consent is widely used. ${ }^{9} 14$ 30-35 In this section, we explore how the concept of minimal risk in research may be applied to clinical practice, particularly regarding guiding clinicians as to what information should be discussed with the patient, regarding particular therapeutics and procedures and health promotion.

Current criteria regarding information provision to the patient generally do not refer to a specific low risk standard. A clinical minimal risk concept comparable with minimal risk in research may assist clinicians in determining the low health risks that need to be discussed and those that do not. A clinical minimal risk concept may be particularly important for family physicians who need to address a wide range of health risk factors beyond clinical procedures and therapies. For example, in prescribing acetylsalicylic acid, which side effects in adult men in addition to gastrointestinal problems should a clinician disclose to the patient? In women who are pregnant or planning pregnancy, must a clinician discuss potential but not yet proven risk of household products such as flame retardants and plasticisers? A clinical minimal risk concept may enhance the patient's informed choice regarding numerous health risks above a minimal risk standard and also save clinician time and resources by providing guidance as to what low-risk factors need to be discussed.

In terms of information regarding clinical procedures, the elements of disclosure and the reasonable patient standard may be suitable particularly for procedures that are deemed the standard of care as recommended in professional practice guidelines or resulting from legal cases. For less common clinical procedures, the subjective standard of disclosure may also be used as it enables clinicians to identify necessary information through communication with the patient as well as the patient's medical and social backgrounds. However, considering the time constraints of clinicians' practice,${ }^{11}$ implementing a clinical minimal 
risk concept could further facilitate the process of determining risks to be discussed and those that require discussion only if raised by the patient. A clinical minimal risk concept may complement currently employed criteria regarding clinicians' risk disclosure in identifying whether a particular risk should be discussed for the patient's informed decision-making.

A clinical minimal risk concept may also have a role in evaluating a variety of risks in the patient's everyday life such as unhealthy diet, substance use or exposure to environmental chemicals, some of which remain uncertain in terms of their potential harm or are not necessarily addressed by clinical guidelines. Needless to say, it is ideal if all risk factors are evaluated at the level of professional bodies, regulatory agencies or society in general. Nonetheless, regulatory responses may not necessarily be timely enough for all potential health risks that are emergent while patients may be anxious about numerous risk factors through the media. Regarding some of these risks, it could be in the patient's best interest to be informed about them particularly if they potentially have significant consequences on health, that is, clearly above minimal risk, and if they are avoidable although uncertainties and the lack of regulatory recommendations should be communicated. Concerning these miscellaneous health risks, a clinical minimal risk concept may be used together with the existing criteria to guide clinicians.

Applying the discussions of absolute versus relative interpretation of minimal risk in research, ${ }^{1} 46{ }^{17-20}$ the relative interpretation of minimal risk may be problematic if a patient lives with high daily risk due to factors such as environment, occupation, recreation activities or health status as it suggests that higher risks in clinical care are minimal risks and thus need not be disclosed. For example, if a person with a serious illness requires a high-risk procedure on a regular basis, the relative interpretation may determine that a different procedure involving a similar level of risk can be performed without disclosing specific risks of the procedure. Likewise, people with a high-risk occupation may not be informed about some risk factors that are communicated to most people. Thus, the absolute interpretation that sets common criteria for everyone may work better as the relative interpretation identifies any risk in a particular person's everyday life as minimal risk for that person, which results in them not being informed about those risks or equally high risks.

Regarding moral justification of the daily life standard, the argument that risks are substitutive ${ }^{18}$ may suit better with clinical procedures as they usually have benefits to the patient as do activities in daily life. Limiting the scope of discussion to a simplified risk-benefit calculation, the relative interpretation of the daily life standard may be morally justifiable in determining what amounts to minimal risk in clinical care. Nevertheless, the risk substitution justification may result in different standards across persons depending on each person's daily life, which is not desirable for guiding clinicians' information provision about particular low risks. Also, the elusiveness of the daily life stand$\operatorname{ard}^{14817}$ should be cautioned against when applied to clinical practice. Moreover, justification based on the claim that daily risks are socially acceptable ${ }^{18}$ may fail to appropriately identify risks that should be discussed with the patient. We contend that the absolute interpretation of the daily life standard is a better model for information provision in clinical care, with some modification required to improve clarity and to ensure exclusion of high risks. An example model may be risks in a healthy adult's daily life at home in a safe neighbourhood.

Moral justification of the routine clinical examination standard based on its adequate exclusion of high risk ${ }^{1}$ and fairness due to less ambiguities in risk assessment ${ }^{8}$ pertains to the clinical context. In fact, the routine clinical examination standard, particularly its absolute interpretation, overlaps with some of the criteria in current clinical practice as routine clinical examinations for healthy persons usually fall under procedures that do not require risk disclosure or explicit consent. Considering the similarities between the routine clinical examination standard and extant clinical criteria for information provision, the routine clinical examination standard appears justifiable in the clinical context for the reasons indicated in the research context. This standard may be useful as it succinctly clarifies a component of existing criteria for clinicians' risk disclosure.

\section{CONCLUSIONS}

Minimal risk in research as a threshold that constitutes a condition for allowing modification to the informed consent process may be extended to help clinicians determine a low risk threshold below which discussion of a particular risk is not required with patients and above which discussion should occur. The absolute interpretation of the daily life standard used in minimal risk in research may be useful with modification in clinical practice. The absolute interpretation of the routine clinical examination standard clarifies a component of extant clinical criteria for risk disclosure. Professional organisations should consider integrating the concept of minimal risk in developing guidelines for facilitating clinicians' information provision to promote the patient's informed choice.

Contributors Both authors contributed substantially to the design, analysis and writing of this manuscript, and have read the final manuscript and take responsibility for its contents.

Funding Supported by Canadian Institutes of Health Research (CIHR) grants RHF100625 and RHF-100626, and the Canadian Institutes of Health Research (CIHR) Training Program.

\section{Competing interests None declared.}

Ethics approval Research ethics review is not required for conceptual research of this nature at our university or in the Tri-Council Policy Statement (of Canada).

Provenance and peer review Not commissioned; externally peer reviewed.

Open Access This is an Open Access article distributed in accordance with the Creative Commons Attribution Non Commercial (CC BY-NC 4.0) license, which permits others to distribute, remix, adapt, build upon this work non-commercially, and license their derivative works on different terms, provided the original work is properly cited and the use is non-commercial. See: http://creativecommons.org/ licenses/by-nc/4.0/

\section{REFERENCES}

1 Kopelman LM. Minimal risk as an international ethical standard in research. J Med Philos 2004;29(3):351-78.

2 Medical Research Council of South Africa. Guidelines on ethics for medical research: general principles. Medical Research Council of South Africa, 2002 (cited 28 March 28 2014);(4). http://www.mrc.ac.za/ethics/ethics.htm

3 Council of Europe. European Treaty Series-No. 195. Additional protocol to the convention on human rights and biomedicine concerning biomedical research. Council of Europe, 2005 (cited 28 March 2014). http://www.coe.int/t/dg3/ healthbioethic/Activities/02_Biomedical_research_en/195\%20Protocole\% 20recherche\%20biomedicale\%20e.pdf

4 Wendler D. Protecting subjects who cannot give consent: toward a better standard for "minimal" risks. Hastings Cent Rep 2005;35(5):37-43.

5 Department of Health and Human Services. Code of federal regulations, title 45 public welfare, part 46 protection of human subjects. US Department of Health \& Human Services, 2009. http://www.hhs.gov/ohrp/humansubjects/guidance/45cfr46. html\#46.116

6 Council for International Organizations of Medical Sciences (CIOMS). International ethical guidelines for biomedical research involving human subjects. CIOMS, 2002. http://www.cioms.ch/publications/layout_guide2002.pdf

7 Canadian Institutes of Health Research, Natural Sciences and Engineering Research Council of Canada, Social Sciences and Humanities Research Council of Canada. Tri-council policy statement: ethical conduct for research involving humans. Government of Canada, 2010. http://www.pre.ethics.gc.ca 
8 Resnik DB. Eliminating the daily life risks standard from the definition of minimal risk. J Med Ethics 2005;31(1):35-8.

9 Appelbaum PS, Berg JW, Lidz CW. Informed consent: legal theory and clinical practice. 2nd edn. New York: Oxford University Press, 2001.

10 Rozovsky FA. Consent to treatment: a practical guide. Boston: Aspen Law \& Business, 1990

11 Moayyeri A, Soltani A, Moosapour $\mathrm{H}$, et al. Evidence-based history taking under "time constraint". J Res Med Sci 2011;16(4):559-64.

12 Levine RJ. Ethics and regulation of clinical research. 2nd edn. New Haven: Yale University Press, 1988.

13 Beauchamp TL. Informed consent: its history, meaning, and present challenges. Camb Q Healthc Ethics 2011;20(4):515-23.

14 Manson NC, O'Neill O. Rethinking informed consent in bioethics. Cambridge: Cambridge University Press, 2007.

15 Miller FG, Wertheimer A. Facing up to paternalism in research ethics. Hastings Cent Rep 2007;37(3):24-34.

16 Yoe CE. Primer on risk analysis decision making under uncertainty. Boca Raton, FL: Taylor \& Francis Group, 2012.

17 Nelson RM. Minimal risk, yet again. J Pediatr 2007;150(6):570-2.

18 Freedman B, Fuks A, Weijer C. In loco parentis. Minimal risk as an ethical threshold for research upon children. Hastings Cent Rxep 1993;23(2):13-19.

19 Tauer CA. Central ethical dilemmas in research involving children. Account Res 2002;9(3-4):127-42.

20 Kopelman LM. Children as research subjects: a dilemma. J Med Philos 2000;25 (6):745-64.

21 Emanuel EJ, Wendler D, Grady C. An ethical framework for biomedical research. In: Emanuel EJ, Lie RK, Grady C, Miller FG, Crouch RA, Wendler D eds. The Oxford textbook of clinical research ethics. New York: Oxford University Press, 2008:123-35.

22 National Commission for the Protection of Human Subjects of Biomedical and Behavioral Research. The Belmont Report: Ethical Principles and Guidelines for the Protection of Human Subjects of Research: DEHW Publication No.(OS)79-12065. 1979.

23 Capron AM. Legal and regulatory standards of informed consent in research. In: Emanuel EJ, Lie RK, Grady C, et al, eds. The Oxford textbook of clinical research ethics. New York: Oxford University Press, 2008:613-22.

24 Drazen JM, Solomon CG, Greene MF. Informed consent and support. N Engl J Med 2013;368(20):1929-31

25 Faden RR, Beauchamp TL, Kass NE. Informed consent, comparative effectiveness, and learning health care. N Engl J Med 2014;370(8):766-8.

26 Macklin R, Shepherd L. Informed consent and standard of care: what must be disclosed. Am J Bioeth 2013;13(12):9-13.

27 Truog RD, Robinson W, Randolph A, et al. Is informed consent always necessary for randomized, controlled trials? N Engl J Med 1999;340(10):804-7.
28 Wendler D. What should be disclosed to research participants? Am J Bioeth 2013;13(12):3-8.

29 ABIM Foundation. American Board of Internal Medicine; ACP-ASIM Foundation. American College of Physicians-American Society of Internal Medicine; European Federation of Internal Medicine. Medical professionalism in the new millennium: a physician charter. Ann Intern Med 2002;136(3):243-6.

30 General Medical Council (United Kingdom). Consent: patients and doctors making decisions together. General Medical Council (United Kingdom), 2008 (cited 31 March 2014). http://www.gmc-uk.org/guidance/ethical_guidance/consent_ guidance_index.asp

31 American Hospital Association. The patient care partnership. American Hospital Association, 2006 (cited 23 May 2012). http://www.aha.org/advocacy-issues/ communicatingpts/pt-care-partnership.shtml

32 Oliveira GHM, Nesbitt GC, Murphy JG. Mayo Clinic medical manual. Boca Raton, FL: Mayo Clinic Scientific Press, 2006.

33 World Medical Association. Medical ethics manual. World Medical Association, 2009 (cited 31 May 2012);(2). http://www.wma.net/en/30publications/ 30ethicsmanual/index.html

34 CMA. CMA code of ethics. Canadian Medical Association, 2004 (cited 1 June 2012).

35 Pozgar GD, Santucci NM. Legal aspects of health care administration. Sudbury, MA: Jones \& Bartlett Learning, 2012.

36 White $C$, Rosoff AJ, LeBlang TR. Informed consent to medical and surgical treatment. In: Sanbar SS, Firestone MH, Fiscina S, et al, eds. Legal medicine. 7th edn. Philadelphia: Mosby Elsevier, 2007:337-45.

37 American College of Physicians. ACP ethics manual. American College of Physicians, 2011 (cited 1 June 2012);(6). http://www.acponline.org/running_practice/ethics/ manual/manual6th.htm\#informed

38 British Medical Association. Consent tool kit. British Medical Association, 2009 (cited 1 June 2012);(5). http://www.bma.org.uk/ethics/consent_and_capacity/ consenttoolkit.jsp

39 Jackson E. Medical law: text, cases, and materials. Oxford: Oxford University Press, 2010.

40 President's Commission for the Study of Ethical Problems in Medicine and Biomedical and Behavioral Research. Making health care decisions: a report on the ethical and legal implications of informed consent in the patient-practitioner relationship. Volume one: report. Washington DC: US Government Printing Office, 1982.

41 American Medical Association. AMA's code of medical ethics. American Medical Association, 2006 (cited 1 June 20120). http://www.ama-assn.org/ama/pub/ physician-resources/medical-ethics/code-medical-ethics.page

42 Bowman D, Spicer J, Iqbal R. Informed consent: a primer for clinical practice. Cambridge: Cambridge University Press, 2012. 